\title{
Proteomic profiling of fast-to-slow muscle transitions during aging
}

\section{Kay Ohlendieck*}

Department of Biology, Muscle Biology Laboratory, National University of Ireland, Maynooth, County Kildare, Ireland

\section{Edited by:}

Ali Mobasheri, The University of Nottingham, UK

\section{Reviewed by:}

Pablo Martin-Vasallo, Universidad de

La Laguna, Spain

Sue Bodine, University of California

Davis, USA

\section{${ }^{*}$ Correspondence:}

Kay Ohlendieck, Department of Biology, Muscle Biology Laboratory, National University of Ireland, Callan Building, Room 2.33, Maynooth,

County Kildare, Ireland.

e-mail:kay.ohlendieck@nuim.ie
Old age is associated with a large spectrum of physical ailments, including muscle wasting. Skeletal muscle degeneration drastically increases the risk of poor balance, frequent falling and impaired mobility in the elderly. In order to identify new therapeutic targets to halt or even reverse age-dependent muscle weakness and improve diagnostic methods to properly evaluate sarcopenia as a common geriatric syndrome, there is an urgent need to establish a reliable biomarker signature of muscle aging. In this respect, mass spectrometry-based proteomics has been successfully applied for studying crude extracts and subcellular fractions from aged animal and human muscle tissues to identify novel aging marker proteins. This review focuses on key physiological and metabolic aspects of sarcopenia, i.e., age-related muscle fiber transitions and metabolic shifts in aging muscle as revealed by proteomics. Over the last decade, proteomic profiling studies have clearly confirmed the idea that sarcopenia is based on a multi-factorial pathophysiology and that a glycolytic-to-oxidative shift occurs in slower-twitching senescent muscles. Both, newly identified protein factors and confirmed alterations in crucial metabolic and contractile elements can now be employed to establish a sarcopenia-specific biomarker signature.

Keywords: biomarker, mass spectrometry, muscle aging, muscle transitions, proteomics, sarcopenia

\section{SARCOPENIA OF OLD AGE}

Although inter-individual differences exist in the onset and severity of the natural aging process of the neuromuscular system, most humans experience an age-dependent loss in skeletal muscle mass accompanied by a considerable decline in contractile strength (Faulkner et al., 2007). In addition to a sedentary lifestyle and an unbalanced diet, other factors can complicate the pathophysiology of progressive muscle wasting in the senescent organism, such as unrelated co-morbidities. Obesity, diabetes, cardiovascular disease, or poor recovery from traumatic injury may affect muscle performance and/or be influenced by changes in the musculature. Epidemiological studies of sarcopenia suggest that nearly half the population over 75 years of age is suffering from muscular weakness leading in severe cases to loss of independence (Berger and Doherty, 2010). The findings from a large number of detailed histological, biochemical, and physiological studies of muscle aging strongly suggest that sarcopenia is due to a multi-factorial pathology. The loss of spinal motor neurons due to apoptosis probably presents one of the most crucial events that eventually leads to a drastic reduction in muscle fiber numbers and size during skeletal muscle aging (Aagaard et al., 2010). The decline in neural organization and an impaired capacity for axonal reinnervation of deinnervated muscle fibers was shown for both aged animals (Edstrom et al., 2007) and senescent humans (Vandervoort, 2002).

In addition to cycles of denervation and faulty reinnervation, age-dependent muscle wasting is associated with the pathophysiological uncoupling between excitation and muscle contraction, impaired muscle protein synthesis, abnormal levels of growth factors and hormones, impaired metabolic pathways, an increased susceptibility to apoptosis, disturbed ion homeostasis, a blunted cellular stress response, and a reduced regenerative capacity (Edstrom et al., 2007). In order to determine a potential hierarchy within these different pathological factors, global genomic, and proteomic investigations have been carried out over the last few years. Molecular genetic studies of sarcopenia have revealed a large number of differentially expressed genes in aged muscle tissue (Tan et al., 2011). It will now be crucial to determine how these age-related gene expression changes translate into an altered abundance and/or post-translational modifications in skeletal muscle proteins. This review outlines the main findings of recent proteomic studies that have focused on physiological and metabolic aspects of sarcopenia, i.e., fast-to-slow transitions and glycolytic-to-oxidative shifts in aging muscle.

\section{PROTEOMICS IN SKELETAL MUSCLE PHYSIOLOGY}

Investigations into the molecular basis of physiological and pathophysiological changes in skeletal muscle tissues have traditionally focused on single or small groups of genes, proteins, or metabolites. With the advance of high-throughput approaches such as genomics, proteomics, and metabolomics, it is now possible to carry out large-scale studies that determine global changes in biomolecules. Mass spectrometry-based proteomics presents an unbiased analytical tool for studying cell biological and physiological phenomena. Proteomics combines standardized biochemical methods in a streamlined approach for tissue extraction, protein separation, protein characterization, and protein identification (Walther and Mann, 2010). In the case of skeletal muscle proteomics, the mass spectrometric identification of altered protein expression patterns has established a large cohort of novel biomarkers associated with myogenesis, physical exercise, 
denervation, stimulation-induced muscle transformation, disuse atrophy, and mechanical unloading, as well as a variety of neuromuscular pathologies (Ohlendieck, 2011).

In addition, an abnormal abundance and/or altered posttranslational modifications were shown to exist in a large number of proteins in senescent skeletal muscle, including components involved in the regulation and maintenance of the excitationcontraction-relaxation cycle, ion homeostasis, and the cellular stress response, as well as both anaerobic and oxidative bioenergetic processes. Since an extensive review of the impact of muscle proteomics on the field of biogerontology has previously been published (Doran et al., 2009), this article will instead focus on a specific aspect of senescent fibers that is highly relevant for skeletal muscle physiology, i.e., the proteomics of muscle plasticity during aging. Relevant proteomic studies on age-related muscle plasticity are listed in Table $\mathbf{1}$. Since proteomic surveys documenting small abundance changes in individual subspecies of muscle proteins may be at variance between different reports on muscle aging, the listed findings in marker proteins do not represent results from specific studies but present a summary of major trends in proteome-wide alterations. This includes mostly regulatory proteins, contractile proteins, metabolic enzymes, and metabolite transporters. Since fiber type shifting and metabolic adaptations represent most likely secondary events during muscle aging, the combination of these novel protein markers can now possibly be used to differentiate early and late stages of sarcopenia for improved diagnostic procedures.

\section{FAST-TO-SLOW TRANSITIONS IN AGED SKELETAL MUSCLE}

Individual skeletal muscles consist of three main types of fibers, slow-oxidative type I fibers, fast oxidative-glycolytic type IIa fibers and fast glycolytic type IIb/x fibers, as well as a variety of hybrid fibers (Schiaffino, 2010). Considerable changes in the fiber type ratio occur as a result of physiological adaptations, in association with many muscular disorders and during the natural aging process. Molecular and cellular modifications in skeletal muscle tissues are reflected by major alterations in protein expression patterns (Gelfi et al., 2011). Slow-to-fast muscle transitions can be typically observed in disuse atrophy, microgravity and extended periods of bed rest. In contrast, endurance exercise, chronic lowfrequency stimulation, hyper-excitability, and aging usually trigger fast-to-slow muscle transformation (Canepari et al., 2010). That proteomics technology is capable of detecting minute changes in the isoform expression pattern of skeletal muscle proteins has previously been demonstrated by the application of fluorescence difference in-gel electrophoresis for the analysis of muscle transitions following chronic electro-stimulation (Donoghue et al., 2007). The same methodology was applied for evaluating potential protein changes in sarcopenia.

During aging, specific force and maximum shortening velocity of muscles are reduced, which is believed to be mostly due to an altered density and property of myosin molecules (Prochniewicz et al., 2007). The pathophysiological shift to a slower muscle phenotype was clearly confirmed by the sub proteomic profiling of the contractile apparatus of aged rat muscle, which revealed a drastic increase in both abundance and phosphorylation levels of slow myosin light chain MLC2 (Gannon et al., 2009). Comprehensive proteomic surveys of crude extracts from aged human and animal muscle agree with the idea of a slower-contracting mode in senescent fiber populations. Key regulatory and contractile elements were shown to exhibit a switch to slower isoforms during aging, including myosin heavy chains, myosin light chains, actin, tropomyosin, and various subunits of the troponin complex (Piec et al., 2005; Gelfi et al., 2006; O'Connell et al., 2007; Doran et al., 2008; Donoghue et al., 2010). Figure 1 outlines the proposed fastto-slow transformation process in aged muscle with respect to contractile proteins. Muscle transitions are probably a secondary occurrence as a consequence of an apoptosis-triggered loss of

Table 1 | Major trends in protein changes during skeletal muscle aging as revealed by mass spectrometry-based proteomics*.

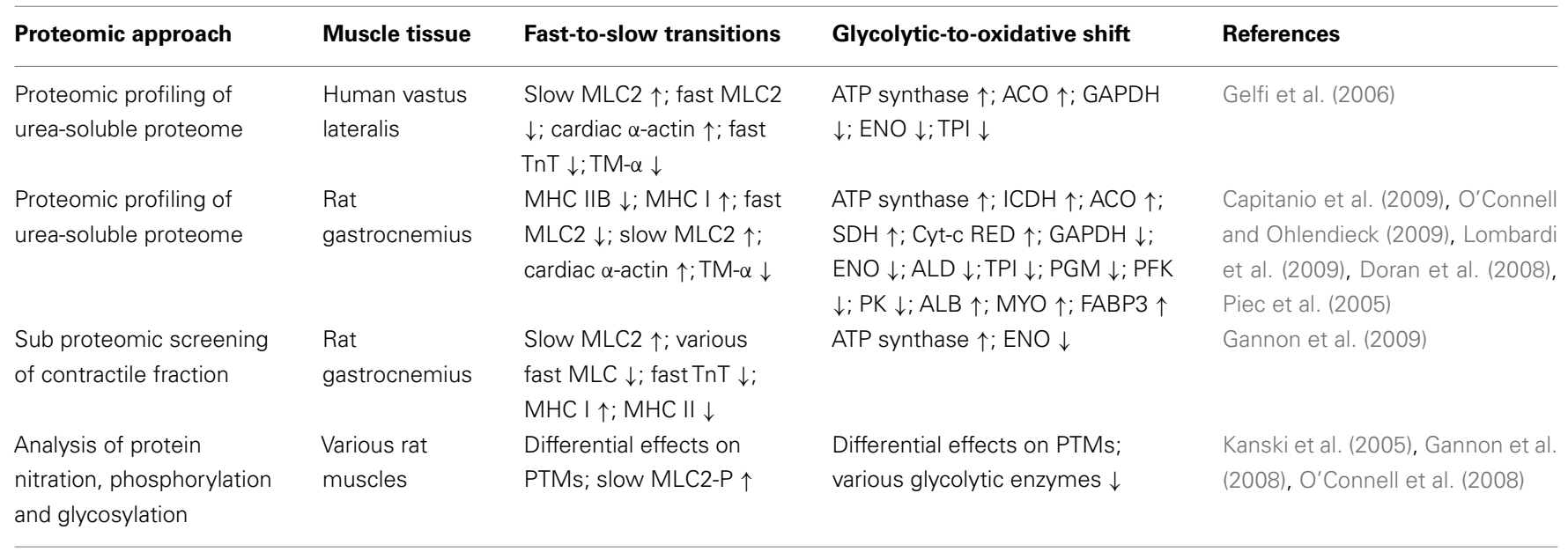

*The table lists markers of the contractile apparatus (MHC, myosin heavy chain; MLC, myosin light chain; TM, tropomyosin; TnT, troponin subunit T) and key enzymes of glycolysis (GAPDH, glyceraldehyde-3-phosphate dehydrogenase; ENO, enolase; ALD, aldolase; TPI, triosephosphate isomerase; PGM, phosphoglucomutase; PFK, phosphofructokinase; $P K$, pyruvate kinase) and oxidative metabolism (ICDH, isocitrate dehydrogenase; $A C O$, aconitase; SDH, succinate dehydrogenase; Cyt-c RED, cytochrome-c reductase; ALB, albumin; MYO, myoglobin; FABP, fatty acid binding-protein). 


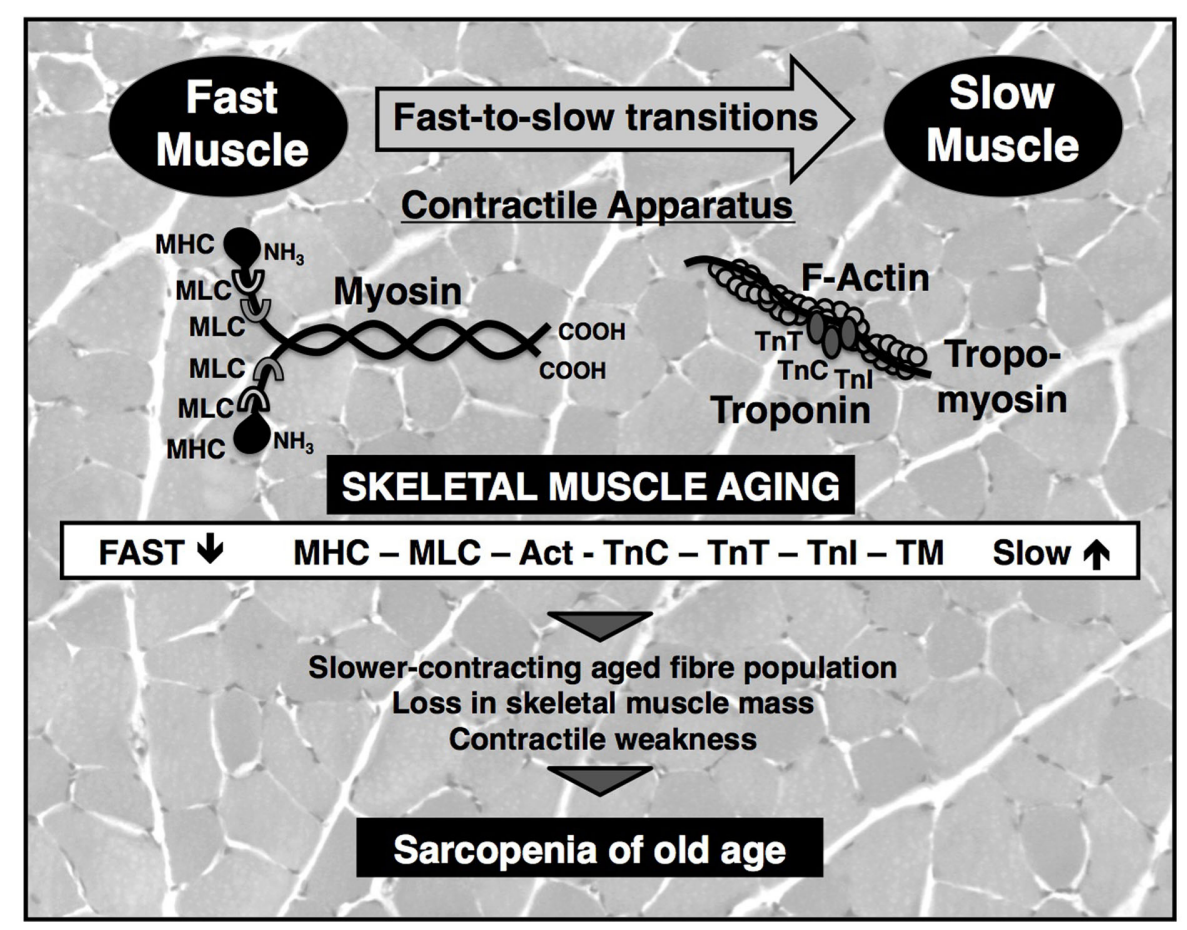

FIGURE 1 | Fast-to-slow muscle transitions during muscle aging as revealed by proteomics. Shown is a diagram of the effect of aging on the main contractile and regulatory proteins of the actomyosin apparatus. Proteomic profiling has clearly established an age-related shift to slower protein isoforms of myosin heavy chain (MHC), myosin light chain (MLC), actin (Act), and tropomyosin (TM), as well as troponin subunits (TnC, TnT, Tnl). Besides the slowing of contractile force, the progressive loss of muscle mass and resulting contractile weakness are the main cause of sarcopenia of old age. The background shows a transverse section of gastrocnemius muscle stained with hematoxylin and eosin. spinal motor neurons, faulty reinnervation mechanisms following denervation and selective atrophy of type II fibers (Vandervoort, 2002; Edstrom et al., 2007; Aagaard et al., 2010).

\section{GLYCOLYTIC-T0-0XIDATIVE SHIFT IN SENESCENT MUSCLE}

Energy for the regulation and maintenance of the excitationcontraction-relaxation cycle is supplied by ATP via anaerobic glycolysis, the phosphocreatine shuttle, the citric acid cycle and oxidative phosphorylation. Carbohydrate and fatty acids constitute major energy substrates during physical exercise and the amino acid pool also interacts with the citric acid cycle in contracting fibers. Under anaerobic conditions pyruvate is converted into lactate, and under aerobic conditions the glycolytic conversion of glucose to pyruvate is followed by the enzymatic reactions of the citric acid cycle and oxidative phosphorylation (Wells et al., 2009). The density of enzymes that are associated with glycolysis, the phosphocreatine shuttle, the citric acid cycle, and oxidative phosphorylation reflect the metabolic status of individual muscles. Although gel electrophoresis-based proteomics is afflicted with various biological and technical problems, it is an ideal analytical tool for studying the abundant and mostly soluble enzymes that constitute the glycolytic system (Ohlendieck, 2010). Proteomics has been successfully applied for studying the catalytic elements associated with glycolysis and shown that their density is drastically altered during development, muscle differentiation, physiological adaptations, and many pathological mechanisms, such as muscular dystrophy or diabetes mellitus. In analogy, proteomic profiling of senescent muscle tissue has confirmed that slowercontracting aged muscle exhibit a glycolytic-to-oxidative shift. This phenomenon is comparable to fast-to-slow transitions in chronic low-frequency stimulated fast muscles, which are characterized by a drastic decrease in glycolytic enzymes and a concomitant increase in mitochondrial markers of oxidative metabolism (Donoghue et al., 2007).

While the expression of glycolytic enzymes such as enolase, triosephosphate isomerase and pyruvate kinase is lower in senescent muscle (Gelfi et al., 2006; Capitanio et al., 2009), mitochondrial enzymes such as succinate dehydrogenase and NADH dehydrogenase are clearly elevated during aging (O'Connell and Ohlendieck, 2009). However, variances in the differential expression of certain glycolytic enzymes exist between human and rodent muscle during aging (Gelfi et al., 2006; Doran et al., 2008; Donoghue et al., 2010). Interestingly, the supramolecular organization of mitochondrial complexes involved in oxidative phosphorylation was shown to be disturbed. Aged mitochondria exhibited lower levels of complex I, complex III, and complex V, but increased amounts of complex II and an unchanged expression of complex IV (Lombardi et al., 2009). A recent proteomic study of calpain-interacting proteins has shown an association between the $\mathrm{Ca}^{2+}$-dependent proteolytic system and ATP synthase and actinin, suggesting a role of calpains in mitochondrial and cytoskeletal dysfunction in sarcopenia (Brule et al., 2010). Age-related 
muscle degeneration was also shown to have a drastic effect on post-translational modifications in numerous metabolic proteins, affecting especially glycosylation, phosphorylation, and tyrosine nitration in glycolytic enzymes (Kanski et al., 2005; Gannon et al., 2008; O'Connell et al., 2008). The diagrammatic presentation in Figure 2 summarizes the involvement of cytosolic and mitochondrial pathways in the proposed glycolytic-to-oxidative shift in aged muscle metabolism. An age-related adaptation of metabolism is most likely a result of lost spinal motor neurons and subsequent faulty reinnervation of denervated muscle fibers, yielding a higher percentage of oxidative type I fibers (Vandervoort, 2002; Edstrom et al., 2007; Aagaard et al., 2010).

\section{CONCLUSION}

Skeletal muscle tissue is highly abundant in the body and plays a central role in metabolism and body movement. Hence, the agedependent loss in muscle mass and function has a severe impact on overall body homeostasis and causes frailty in aged individuals. The frailty syndrome is increasingly recognized as an extreme risk indicator of adverse health outcomes in the elderly (Evans et al., 2010). This warrants detailed molecular and cellular studies into the molecular pathogenesis of metabolic and contractile dysregulation in the aged neuromuscular system. Mass spectrometrybased proteomics has clearly confirmed a fast-to-slow contractile transformation process and a glycolytic-to-oxidative metabolic shift during skeletal muscle aging. From a pathophysiological point of view, it is unlikely that fiber type shifting or bioenergetic changes are causative factors of sarcopenia, but rather a consequence of muscle wasting. Primary factors with an unknown pathological hierarchy are proposed to be loss of motor neurons, chronic inflammation, insulin resistance, disuse-related muscular atrophy, decreased levels of essential growth hormones, a reduced regenerative capacity, and various nutritional deficiencies with advancing age (Evans, 2010). Many histological studies indicate that a transition in fiber composition occurs during aging with a higher fiber type I to fiber type II ratio in senescent muscle tissue, probably based on selective atrophy of fast-twitching fiber populations (Vandervoort, 2002). Although there is no consensus on this aspect of a fiber-selective degradation process during the molecular pathogenesis of sarcopenia, proteomic findings clearly agree with a general reduction in the fast fiber population in the elderly.

As reviewed by Berger and Doherty (2010), after the fifth decade a $2 \%$ reduction in muscle mass per year is observed in many humans, which has a serious impact on the steadily increasing number of aged members of society. Thus, to prevent the loss of independence due to severe age-related impairments of the neuromuscular system, the urgent implementation of resistance training programs (Mangione et al., 2010) combined with a protein-rich and balanced diet (Rolland et al., 2011) is needed to promote healthy aging. For the proper differential diagnosis of sarcopenia of old age and the swift evaluation of novel treatment regimes, the

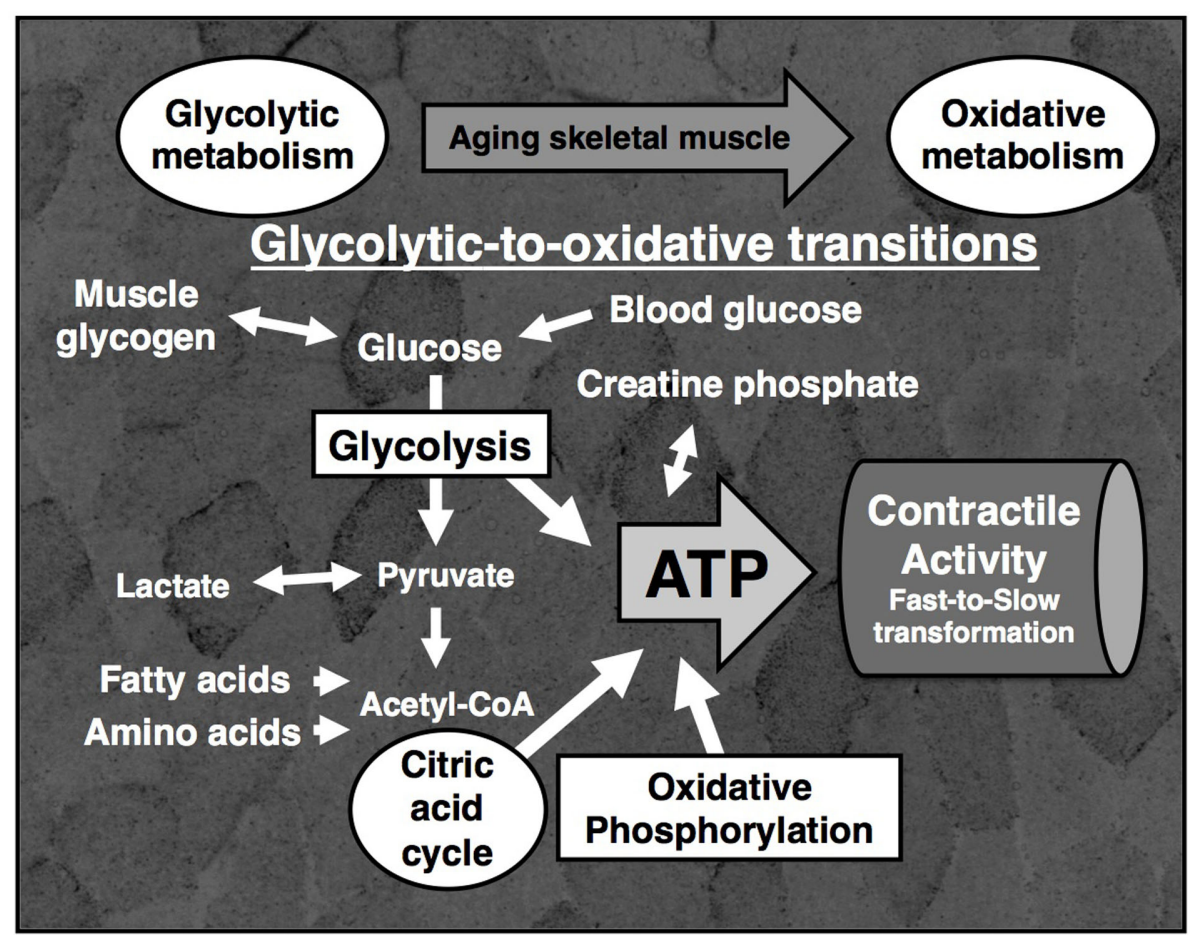

FIGURE 2 | Glycolytic-to-oxidative shift during muscle aging as revealed by proteomics. Shown is a diagram of the main bioenergetic pathways for the provision of adenosine triphosphate (ATP) for the contractile activity of skeletal muscle fibers including glycolysis, the creatine phosphate shuttle, the citric acid cycle and oxidative phosphorylation. Proteomic profiling has clearly established an increase in mitochondrial enzymes and concomitant decrease in glycolytic enzymes during the fast-to-slow transformation process in aging skeletal muscle tissue. The background shows a transverse section of gastrocnemius muscle that was histochemically stained for the presence of the mitochondrial marker enzyme succinate dehydrogenase. 
availability of reliable biomarkers is essential. In order to catalog and characterize as many new indicators as possible, future biochemical studies should be more comprehensive with respect to integral membrane proteins and low-abundance proteins, which are currently underestimated in proteome-wide surveys. This will require the application of modified proteomic techniques involving improved protein separation methods, enhanced protein

\section{REFERENCES}

Aagaard, P., Suetta, C., Caserotti, P., Magnusson, S. P., and Kjaer, M. (2010). Role of the nervous system in sarcopenia and muscle atrophy with aging: strength training as a countermeasure. Scand. J. Med. Sci. Sports 20, 49-64.

Berger, M. J., and Doherty, T. J. (2010). Sarcopenia: prevalence, mechanisms, and functional consequences. Interdiscip. Top. Gerontol. 37, 94-114.

Brule, C., Dargelos, E., Diallo, R., Listrat, A., Bechet, D., Cottin, P., and Poussard, S. (2010). Proteomic study of calpain interacting proteins during skeletal muscle aging. Biochimie 92, 1923-1933.

Canepari, M., Pellegrino, M. A., D'Antona, G., and Bottinelli, R. (2010). Skeletal muscle fibre diversity and the underlying mechanisms. Acta Physiol. 199, 465-476.

Capitanio, D., Vasso, M., Fania, C., Moriggi, M., Vigano, A., Procacci, P., Magnaghi, V., and Gelfi, C. (2009). Comparative proteomic profile of rat sciatic nerve and gastrocnemius muscle tissues in ageing by 2-D DIGE. Proteomics 9, 2004-2020.

Donoghue, P., Doran, P., Wynne, K., Pedersen, K., Dunn, M. J., and Ohlendieck, K. (2007). Proteomic profiling of chronic low-frequency stimulated fast muscle. Proteomics 7 , 3417-3430.

Donoghue, P., Staunton, L., Mullen, E., Manning, G., and Ohlendieck, K. (2010). DIGE analysis of rat skeletal muscle proteins using nonionic detergent phase extraction of young adult versus aged gastrocnemius tissue. J. Proteomics 73, 1441-1453.

Doran, P., Donoghue, P., O'Connell, K., Gannon, J., and Ohlendieck, K. (2009). Proteomics of skeletal muscle aging. Proteomics 9, 989-1003.

Doran, P., O'Connell, K., Gannon, J., Kavanagh, M., and Ohlendieck, K. (2008). Opposite pathobiochemical fate of pyruvate kinase and adenylate kinase in aged rat skeletal muscle as revealed by proteomic DIGE analysis. Proteomics 8, 364-377.

Edstrom, E., Altun, M., Bergman, E., Johnson, H., Kullberg, S., RamirezLeon, V., and Ulfhake, B. (2007). Factors contributing to neuromuscular impairment and sarcopenia during aging. Physiol. Behav. 92, 129-135.

Evans, W. J. (2010). Skeletal muscle loss: cachexia, sarcopenia, and inactivity. Am. J. Clin. Nutr. 91, 1123S-1127S

Evans, W. J., Paolisso, G., Abbatecola, A. M., Corsonello, A., Bustacchini, S., Strollo, F., and Lattanzio, F. (2010). Frailty and muscle metabolism dysregulation in the elderly. Biogerontology 11, 527-536.

Faulkner, J. A., Larkin, L. M., Claflin, D. R., and Brooks, S. V. (2007). Age-related changes in the structure and function of skeletal muscles. Clin. Exp. Pharmacol. Physiol. 34, 1091-1096.

Gannon, J., Doran, P., Kirwan, A., and Ohlendieck, K. (2009). Drastic increase of myosin light chain MLC2 in senescent skeletal muscle indicates fast-to-slow fibre transition in sarcopenia of old age. Eur. J. Cell Biol. $88,685-700$.

Gannon, J., Staunton, L., O’Connell, K., Doran, P., and Ohlendieck, K. (2008). Phosphoproteomic analysis of aged skeletal muscle. Int. J. Mol. Med. 22, 33-42.

Gelfi, C., Vasso, M., and Cerretelli, P. (2011). Diversity of human skeletalmuscle in health and disease: contribution of proteomics. J. Proteomics 74, 774-795.

Gelfi, C., Vigano, A., Ripamonti, M., Pontoglio, A., Begum, S., Pellegrino, M. A., Grassi, B., Bottinelli, R., Wait, R., and Cerretelli, P. (2006). The human muscle proteome in aging. J. Proteome Res. 5, 1344-1353.

Kanski, J., Hong, S. J., and Schoneich, C. (2005). Proteomic analysis of protein nitration in aging

digestion approaches and more sensitive mass spectrometric analyses.

\section{ACKNOWLEDGMENTS}

This work was supported by project grants from the Irish Health Research Board, Science Foundation Ireland, the Higher Education Authority, and Muscular Dystrophy Ireland.

skeletal muscle and identification of nitrotyrosine-containing sequences in vivo by nanoelectrospray ionization tandem mass spectr ometry. J. Biol. Chem. 280, 24261-24266.

Lombardi, A., Silvestri, E., Cioffi, F, Senese, R., Lanni, A., Goglia, F., de Lange, P., and Moreno, M. (2009). Defining the transcriptomic and proteomic profiles of rat ageing skeletal muscle by the use of a cDNA array, 2D- and Blue native-PAGE approach. J. Proteomics 72, 708-721.

Mangione, K. K., Miller, A. H., and Naughton, I. V. (2010). Cochrane review: improving physical function and performance with progressive resistance strength training in older adults. Phys. Ther. 90, 1711-1715.

O’Connell, K., Doran, P., Gannon, J., and Ohlendieck, K. (2008). Lectinbased proteomic profiling of aged skeletal muscle: decreased pyruvate kinase isozyme M1 exhibits drastically increased levels of $\mathrm{N}$ glycosylation. Eur. J. Cell Biol. 87, 793-805.

O'Connell, K., Gannon, J., Doran, P., and Ohlendieck, K. (2007). Proteomic profiling reveals a severely perturbed protein expression pattern in aged skeletal muscle. Int. J. Mol. Med. 20, 145-153.

O'Connell, K., and Ohlendieck, K. (2009). Proteomic DIGE analysis of the mitochondria-enriched fraction from aged rat skeletal muscle. Proteomics 9, 5509-5524.

Ohlendieck, K. (2010). Proteomics of skeletal muscle glycolysis. Biochim. Biophys. Acta 1804, 2089-2101.

Ohlendieck, K. (2011). Skeletal muscle proteomics: current approaches, technical challenges and emerging techniques. Skelet. Muscle 1, 6.

Piec, I., Listrat, A., Alliot, J., Chambon, C., Taylor, R. G., and Bechet, D. (2005). Differential proteome analysis of aging in rat skeletal muscle. FASEB J. 19, 1143-1145.

Prochniewicz, E., Thompson, L. V., and Thomas, D. D. (2007). Age-related decline in actomyosin structure and function. Exp. Gerontol. 42, 931-938.

Rolland, Y., Dupuy, C., Abellan van Kan, G., Gillette, S., and Vellas, B. (2011). Treatment strategies for sarcopenia and frailty. Med. Clin. North Am. 95, $427-438$.

Schiaffino, S. (2010). Fibre types in skeletal muscle: a personal account. Acta Physiol. 199, 451-463.

Tan, L. J., Liu, S. L., Lei, S. F., Papasian, C. J., and Deng, H. W. (2011). Molecular genetic studies of gene identification for sarcopenia. Hum. Genet. doi: 10.1007/s00439-011-1040-7. [Epub ahead of print].

Vandervoort, A. A. (2002). Aging of the human neuromuscular system. Muscle Nerve 25, 17-25.

Walther, T. C., and Mann, M. (2010). Mass spectrometry-based proteomics in cell biology. J. Cell Biol. 190, 491-500.

Wells, G. D., Selvadurai, H., and Tein I. (2009). Bioenergetic provision of energy for muscular activity. Paediatr. Respir. Rev. 10, 83-90.

Conflict of Interest Statement: The author declares that the research was conducted in the absence of any commercial or financial relationships that could be construed as a potential conflict of interest.

Received: 03 November 2011; accepted: 03 December 2011; published online: 26 December 2011.

Citation: Ohlendieck K (2011) Proteomic profiling of fast-to-slow muscle transitions during aging. Front. Physio. 2:105. doi: 10.3389/fphys.2011.00105

This article was submitted to Frontiers in Striated Muscle Physiology, a specialty of Frontiers in Physiology.

Copyright $\odot 2011$ Ohlendieck. This is an open-access article distributed under the terms of the Creative Commons Attribution Non Commercial License, which permits non-commercial use, distribution, and reproduction in other forums, provided the original authors and source are credited. 